\title{
From coal to nano carbon to cross blood brain barrier to reach neuron
}

\author{
Sabyasachi Sarkar
}

Center for Healthcare Science and Technology, Nanoscience and Artificial Leaf Laboratory ( Downing Hall), Indian Institute of Engineering Science and Technology, Shibpur ( IIESTS), Botanic Garden, Howrah 711103, West Bengal, India

Graphene oxide, (GO), formed under metamorphism in coalification of plants in the low grade coal is extracted by leaching with cold nitric acid. Similar green extraction of GO is followed from wood charcoal. Such GO sheets are thermally not very stable and on aging is fragmented into small spherical shape (Figure 1). These are highly soluble and are larger than graphene quantum dot and under varied stimuli get folded like fisted form. These can entrap tetraphenylporphyrin (TPP), a model chemical for photodynamic therapy (PDT) in phosphate buffered saline (PBS). Similarly the drug Donepezil, used in Alzheimer gets entrapped by GO. The clenched fist form of corrugated $\mathrm{GO}$ is related to the $\mathrm{pH}$ dependent epoxide dominated structure as proposed by Hofmann and Holst's model and the open structure may be dominated by hydroxyl groups (enol) following Ruess's model .Such pH dependent inter-conversion uniquely provide GO to act as a cargo in carrying a drug molecule and its release under physio-compatible conditions. These became soluble in water and were used in in vivo imaging of the entire life cycle of Drosophila melanogester. We also explored the utility of these nano carbons in plant kingdom. Based on such usefulness we explored the utility of fisted form of water soluble carbon nano onion (wsCNO) to probe brain in the light of the existence of blood brain barrier [1,2].

We are delighted to report that wsCNO have crossed through the blood brain barrier (BBB) and entered the brain without causing any perfusion (Figure 2). This raises immense possibilities for drug delivery in the brain. The assorted sizes of wsCNO may carry and de-load drug molecule accommodated inside like Trojan horse and these can readily be removed from the site after the delivery. A process of "close and open sesame" is a smart structural changes experienced by these nano carbons effective under application of external stimuli. (a)

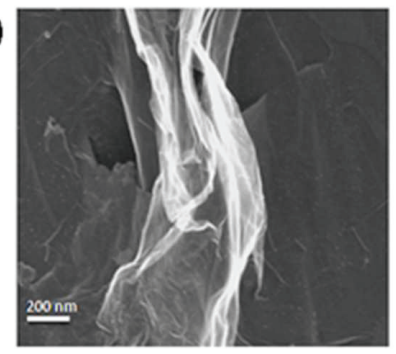

(b)

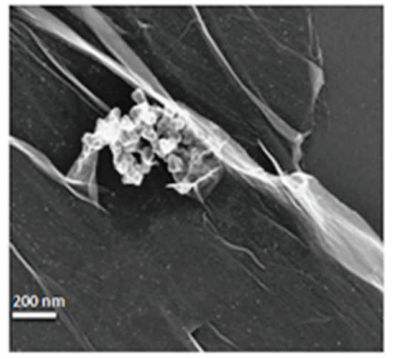

Figure 1: SEM images of GO prepared from coal, (a) freshly prepared, b) aged sample transforming to spherical shape
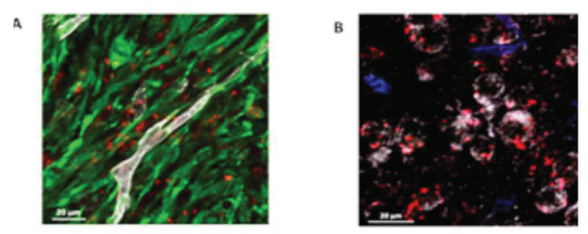

Figure 2: (a) Tumor cells (green), blood vessel (white), wsCNO (red): (b) NeuN antibody stain labeled neurons(white), wsCNO (red) reached the site of neurons

\section{References}

1.B. Pakhira, S. Ghosh, S. Maity, D.N. Sangeetha, A. Laha, A. Allam and S. Sarkar, RSC. Adv. 05,(2015),89076-89082.

2.B. Pakhira, M. Ghosh, A. Allam and S. Sarkar, RSC Adv., 06,(2016), 29779-29782 